\title{
The therapeutic effect of the BRD4-degrading PROTAC A1874 in human colon cancer cells
}

\author{
An-cheng Qin ${ }^{1}$, Hua Jin², Yu Song ${ }^{3}$, Yun Gao ${ }^{4}$, Yi-Fan Chen ${ }^{1}$, Li-na Zhou ${ }^{5}$, Shu-sheng Wang ${ }^{6}$ and Xing-sheng Lu
}

\begin{abstract}
A1874 is a novel BRD4-degrading proteolysis targeting chimera (PROTAC). In primary colon cancer cells and established HCT116 cells, A1874 potently inhibited cell viability, proliferation, cell cycle progression, as well as cell migration and invasion. The BRD4-degrading PROTAC was able to induce caspase and apoptosis activation in colon cancer cells. Furthermore, A1874-induced degradation of BRD4 protein and downregulated BRD-dependent genes ( $c$ Myc, BCl-2, and cyclin D1) in colon cancer cells. Significantly, A1874-induced anti-colon cancer cell activity was more potent than the known BRD4 inhibitors (JQ1, CPI203, and I-BET151). In BRD4-knockout colon cancer cells A1874 remained cytotoxic, indicating the existence of BRD4-independent mechanisms. In addition to BRD4 degradation, A1874 cytotoxicity in colon cancer cells was also associated with p53 protein stabilization and reactive oxygen species production. Importantly, the antioxidant N-acetyl-cysteine and the p53 inhibitor pifithrin-a attenuated A1874-induced cell death and apoptosis in colon cancer cells. In vivo, A1874 oral administration potently inhibited colon cancer xenograft growth in severe combined immuno-deficient mice. BRD4 degradation and p53 protein elevation, as well as apoptosis induction and oxidative stress were detected in A1874-treated colon cancer tissues. Together, A1874 inhibits colon cancer cell growth through both BRD4-dependent and -independent mechanisms.
\end{abstract}

\section{Introduction}

Colon cancer has become a common malignancy and a global health issue ${ }^{1,2}$, causing significant cancer-related human mortalities each year ${ }^{3,4}$. Current clinical treatments, including chemotherapy, surgery, radiation, and/or molecularly targeted therapies ${ }^{1,5,6}$, along with the advanced early screen and diagnosis techniques, have significantly improved the prognosis and five-year overall survival of colon cancer patients ${ }^{1,5,6}$. Yet the prognosis for the advanced, metastatic and recurrent colon cancer patients remains poor ${ }^{1}$. There is an urgent need to further

Correspondence: Li-na Zhou (zhoulinaks@163.com) or Shu-

sheng Wang (drwangsszjg@163.com) or Xing-sheng Lu (drluxsslyy@163.com)

${ }^{1}$ Department of Hepatobiliary Surgery, Suzhou Municipal Hospital Affiliated to

Nanjing Medical University, Suzhou, China

${ }^{2}$ The Child Health Care Department, Suzhou Ninth People's Hospital, Suzhou,

China

Full list of author information is available at the end of the article

These authors contributed equally: An-sheng Qin, Hua Jin, Yu Song and

Yun Gao

Edited by S. Tait explore the underlying pathological mechanisms of colon cancer development and progression ${ }^{1,5,6}$.

Bromodomain-containing protein 4 (BRD4) is an extensively studied BET (bromodomain and extraterminal domain) family protein, that provides a promising therapeutic target for colon cancer ${ }^{7}$. BRD4 directly binds to acetylated histones and plays an essential role in regulating epigenetic processes ${ }^{8-10}$. In the process of mitosis, BRD4 is required for chromatin structure formation in the daughter cells ${ }^{9,11}$. Furthermore, BRD4 is important for transcription elongation and expression of key oncogenic genes including Bcl-2 and c-Myc. BRD4 associates with positive transcription elongation factor $\mathrm{b}$ (P-TEFb) to phosphorylate RNA polymerase II in proliferating cells ${ }^{9,11}$. Recent studies have proposed BRD4 as a novel oncogene as it is overexpressed in colon cancer ${ }^{12}$ and many other malignancies ${ }^{8,11}$.

Multiple small-molecule inhibitors of BRD4 have been developed, showing promising anticancer results in experimental and clinical cancer studies ${ }^{8,11,13}$. However,

\section{(c) The Author(s) 2020}

(c) (i) Open Access This article is licensed under a Creative Commons Attribution 4.0 International License, which permits use, sharing, adaptation, distribution and reproduction cc) in any medium or format, as long as you give appropriate credit to the original author(s) and the source, provide a link to the Creative Commons license, and indicate if changes were made. The images or other third party material in this article are included in the article's Creative Commons license, unless indicated otherwise in a credit line to the material. If material is not included in the article's Creative Commons license and your intended use is not permitted by statutory regulation or exceeds the permitted use, you will need to obtain permission directly from the copyright holder. To view a copy of this license, visit http://creativecommons.org/licenses/by/4.0/. 
BRD4 inhibition results in feedback elevation of BRD4 protein in human cancer cells, leading to weak antiproliferative activity and less apoptosis induction ${ }^{8,11,13}$. A recent study identified A1874 as a BRD4-targeting mouse double minute 2 homolog (MDM2)-based proteolysis targeting chimera (PROTAC) ${ }^{14}$.

Unlike the BRD4 inhibitors, A1874 can lead to a robust and sustained BRD4 protein degradation through ubiquitin system ${ }^{14}$, causing profound inhibition of BRD4dependent cancers ${ }^{14}$. In this study, we tested the potential anticancer activity and underlying signaling mechanisms of A1874 against human colon cancer cells.

\section{Materials and methods}

\section{Chemicals, reagents, and antibodies}

A1874 was obtained from Hanxiang BioTech (Shanghai, China). Puromycin, JQ1, CPI203, I-BET151, N-acetylcysteine (NAC) and pifithrin- $\alpha$, polybrene and CCK- 8 were purchased from Sigma-Aldrich (St. Louis, MO). All cell culture reagents were provided by Hyclone Co. (Logan, UT). Antibodies for c-Myc (\#9402), Cyclin D1 (\#2922), BRD4 (\#13440), Bcl-2 (\#15707), Erk1/2 (\#9102), p53 (\#9282), cleaved-caspase-3 (\#9664), cleaved-caspase-9 (\#20750), cleaved-poly (ADP-ribose) polymerase (PARP) (\#5625), Bcl-2 (\#15707) and $\beta$-tubulin (\#15115) were purchased from Cell Signaling Tech (Beverly, MA). Caspase inhibitors, z-VAD-fmk and z-DEVD-fmk, were provided by Thermo-Fisher (Shanghai, China). Lipofectamine 2000, TUNEL (terminal deoxynucleotidyl transferase dUTP nick end labeling), Annexin V and propidium iodide (PI) were purchased from ThermoFisher Invitrogen (Carlsbad, CA). The BrdU ELISA kit was provided by Roche Diagnostics (Basel, Switzerland).

\section{Cell culture}

The primary human colon cancer cells from four written-informed consent primary colon cancer patients, pCan $1 / 2 / 3 / 4$, and primary human colon epithelial cells from two healthy donors, pEpi1/2, were provided by Dr. $\mathrm{Lu}$ at Nanjing Medical University ${ }^{15-17}$. The primary human cells were cultured in the described medium ${ }^{15,18}$. The established HCT116 colon cancer cells were also provided by $\mathrm{Dr}$. $\mathrm{Lu}^{15-17}$. All the established and primary cells were subjected to routine mycoplasma and microbial contamination examination every $2-3$ months. To confirm the genotype of the cells, STR (short tandem repeat) profiling, population doubling time, and cell morphology were regularly checked. The protocols of this study were approved by Ethic Committee of Nanjing Medical University, according to Declaration of Helsinki.

\section{Cell viability assay}

The viable colon cancer cells or colon epithelial cells were seeded into 96 -well plates at $5 \times 10^{3}$ cells per well.
CCK- 8 kit was utilized to determine cell viability, with CCK-8 optical densities (ODs) recorded at $550 \mathrm{~nm}$.

\section{Colony formation}

Colon cancer cells with the applied A1874 treatment were re-suspended in $1 \mathrm{~mL}$ of DMEM with $0.5 \%$ agar (Sigma). Cells were then added onto the pre-solidified 10$\mathrm{cm}$ cell culture dish. Medium was renewed every 2 days for a total of five rounds. Afterwards, the remaining cell colonies were stained and manually counted.

\section{BrdU ELISA}

Cells were seeded into 96 -well plates at $5 \times 10^{3}$ cells per well. With the applied A1874 treatment, BrdU incorporation was tested by a BrdU ELISA kit according to the attached protocol. BrdU ELISA absorbance was recorded at $405 \mathrm{~nm}$.

\section{EdU staining}

The viable colon cancer cells were seeded into 12-well plates $\left(8 \times 10^{4}\right.$ cells per well). With the applied A1874 treatment, EdU (5-ethynyl-20-deoxyuridine) staining assay was performed as described elsewhere ${ }^{19,20}$. EdU percentages (EdU vs. DAPI, \%) of 1000 cells per treatment in five random views (under a fluorescence microscope at $1 \times 100$ magnification) were recorded.

\section{Cell migration and invasion assays}

Colon cancer cells $\left(3 \times 10^{4}\right.$ cells per chamber, in serumfree medium) were seeded onto the "Transwell" chambers: (8- $\mu \mathrm{m}$ pore size, Corning Costar, Shanghai, China). Complete medium containing 10\% FBS was added to the lower chambers ${ }^{21}$. After incubation for $24 \mathrm{~h}$, the migrated cells were fixed and stained. For cell invasion assays, chambers were always coated with Matrigel (Sigma) ${ }^{22}$. Average number of migrated/invaded cells in five random views per treatment were recorded.

\section{Cell cycle FACS}

The primary human colon cancer cells were seeded into six-well plate at $2 \times 10^{5}$ cells per well. With the applied A1874 treatment, cells were washed, fixed, and incubated with DNase-free RNase and PI. Cell cycle distribution was recorded by using a FACSCalilur machine (BD Biosciences, Shanghai, China).

\section{Caspase activity}

The viable colon cancer cells were seeded into six-well plates (at $2 \times 10^{5}$ cells per well). Following treatment, $30 \mu \mathrm{g}$ of cytosolic extract lysates (per treatment) were added to the caspase assay buffer ${ }^{23}$ together with the applied 7amido-4-(trifluoromethyl)-coumarin (AFC)-conjugated caspase-3/-8/-9 substrate $^{23}$. The AFC fluorescence intensity was tested under a Fluoroskan machine ${ }^{23}$. 


\section{Mitochondrial depolarization}

JC-1 dye can aggregate into mitochondria and form green monomers in the apoptotic cells with mitochondrial depolarization ${ }^{24}$. The viable colon cancer cells were seeded into six-well plates (at $2 \times 10^{5}$ cells per well). Following treatment, cells were incubated with JC-1 $(5.0 \mu \mathrm{g} /$ $\mathrm{mL}$ ). JC-1 intensity was tested immediately under a fluorescence spectrofluorometer at $488 \mathrm{~nm}$. The representative merged JC-1 images were presented as well.

\section{Annexin V-PI-FACS}

The primary human colon cancer cells were seeded into six-well plate at $2 \times 10^{5}$ cells per well. Forty-eight hours after the applied A1874 treatment, cells were harvested, washed, and incubated with Annexin V and PI $(10 \mu \mathrm{g} / \mathrm{mL}$ each). Afterwards, cells were gated under a FACSCalibur machine (BD Biosciences). The percentage of Annexin Vpositive cells was always recorded.

\section{TUNEL staining}

The viable colon cancer cells or colon epithelial cells were seeded into 12 -well plates $\left(8 \times 10^{4}\right.$ cells per well). Forty-eight hours after the applied A1874 treatment, cells were incubated with both TUNEL and DAPI dyes. The nuclear TUNEL percentage (TUNEL vs. DAPI, \%) of 1000 cells per treatment in five random views (under a fluorescence microscope at $1 \times 100$ magnification) was recorded.

\section{Trypan blue assay}

Colon cancer cells were seeded into 12-well plates $(8 \times$ $10^{4}$ cells per well). Seventy-two hours after the applied A1874 treatment, Trypan blue dye was added. Its ratio was recorded by using an automatic cell counter.

\section{Western blotting}

Colon cancer cells were seeded into six-well plate at $2 \times$ $10^{5}$ cells per well. After the applied A1874 treatment, cells were incubated with the described lysis buffer ${ }^{25}$. Total protein lysates (30 $\mu$ g per treatment in each lane) were analyzed. Western blotting protocols were described previously $^{26}$. Data quantification was done through the ImageJ software (NIH).

\section{Quantitative real-time PCR (qPCR)}

Colon cancer cells were seeded into six-well plate at $2 \times$ $10^{5}$ cells per well. Following the applied treatment, TRIzol reagents were utilized to extract total RNA, and the latter was converted into complementary DNA (cDNA). qPCR was carried out using a SYBR Premix Ex Taq ${ }^{\mathrm{TM}}$ kit (TaKaRa, Tokyo, Japan) under the ABI Prism 7500 Fast Real-Time PCR system. GAPDH was always used as the reference gene and the internal control. Quantification was performed with the $2^{-\Delta \Delta C t}$ method. The RNA primer sequences employed in this study were from Dr. Zhu at Soochow University ${ }^{27}$.

\section{Reactive oxygen species (ROS) assay}

Colon cancer cells were seeded into six-well plate at $2 \times$ $10^{5}$ cells per well. After the applied A1874 treatment, cells were stained with CellROX dye (Beyotime, Wuxi, China) and thereafter tested via a fluorescence microscopy.

\section{GSH/GSSG ratio}

Reduced glutathione (GSH) is a vital ROS scavenger in human cells. Its ratio with the oxidized disulfide form glutathione (GSSG) was tested as a quantitative indicator of oxidative stress intensity ${ }^{28}$. Colon cancer cells were seeded into six-well plate at $2 \times 10^{5}$ cells per well. With the applied A1874 treatment, cells were lysed. The GSH/ GSSG ratio was measured using a GSH/GSSG assay kit (Beyotime). GSH/GSSG ratio in human tissues was tested similarly.

\section{Assaying DNA breaks}

The viable colon cancer cells were seeded into 96-well plates at $5 \times 10^{3}$ cells per well. Following the applied A1874 treatment, a single strand DNA (ssDNA) ELISA kit (Roche, Shanghai, China) was utilized to test DNA breaks. The ssDNA ELISA absorbance was tested by $405 \mathrm{~nm}$.

\section{Exogenous BRD4 overexpression}

The pSUPER-puro-GFP expression vector, containing the mutant BRD4 at the MDM2 binding sites, was provided by Dr. Zhao at Soochow University ${ }^{29}$. It was transfected to HEK-293 cells together with viral packaging proteins (VSVG and Hit-60) (provided by Dr. Zhao ${ }^{29}$ ) to generate BRD4-expressinglentivirus. Virus was then enriched, filtered and added to cultured colon cancer cells (in polybrene-containing complete medium), and stable cells selected by puromycin. Exogenous BRD4 overexpression was verified by Western blotting.

\section{BRD4 knockout}

A CRISPR/Cas9-BRD4-knockout (KO) plasmid (with puromycin selection gene, from Dr. Zhao at Soochow University $^{29}$ ) was transfected into primary colon cancer cells via a Lipofectamine 2000 (Thermo-Fisher Invitrogen) protocol. Cells were distributed to 96-well plates to establish single cells and were subjected to BRD4-KO screening (qPCR). Stable cells were further selected by puromycin for 4-5 passages. BRD4 KO in the stable cells was always verified by Western blotting.

\section{Tumor xenografts}

The severe combined immuno-deficient (SCID) mice (5-6 week old, 18-19g weight, all female) were purchased from the Animal Facility of Soochow University (Suzhou, 
China). The primary pCan 1 colon cancer cells $\left(8 \times 10^{6}\right.$ cells per mouse) were subcutaneously (s.c.) injected to the flanks of SCID mice. Within three weeks the tumors reached the average volume of $100 \mathrm{~mm}^{3}$. Tumor-bearing mice were then randomly assigned into two groups (nine mice per group $/ n=9$ ). Mice were then treated with A1874 or the vehicle control. Tumor volumes were recorded using the described formula ${ }^{30}$. Estimated daily tumor growth (in $\mathrm{mm}^{3}$ per day) was calculated as described $^{16}$. All animal studies were in accordance with regulations of the Institutional Animal Care and Use Committee and Ethics Committee of Nanjing Medical University (Nanjing, China).

\section{Statistical analysis}

The investigators were blinded to the group allocation during all experiments. In vitro experiments were repeated at least three times. Data were presented as mean \pm standard deviation (SD). Statistics analyses were carried out through one-way ANOVA with the Scheffe' and Tukey Test (SPSS 23.0, SPSS, Chicago, IL). To determine significance between two treatment groups, the unpaired $t$ test was used (Excel 2007). Significance was determined as $P<0.05$. All the protocols of this study were approved by Ethics Committee of Nanjing Medical University.

\section{Results}

A1874 inhibits colon cancer cell growth, proliferation, cell cycle progression, migration, and invasion

To examine the anti-proliferative activity of A1874, primary human colon cancer cells, pCan1, were cultured in FBS-containing complete medium and treated with increasing concentrations (5-500 nM) of A1874. A CCK-8 assay was carried out to test cell viability. As shown (Fig. 1a), A1874 decreased cell viability in pCan1 cells in a concentration-dependent manner. There was a significant reduction in viability following treatment with $25-500 \mathrm{nM}$ of A1874 (Fig. 1a), whereas the lower concentration (5 nM) was ineffective (Fig. 1a). The BRD4-degrading PROTAC displayed a time-dependent response in inhibiting pCan1 cell viability (Fig. 1b). A1874 (25-500 nM) required at least $48 \mathrm{~h}$ to exert significant anti-survival activity (Fig. 1a). Furthermore, a colony formation assay (Fig. 1c) demonstrated that A1874 (25-500 nM) potently decreased the number of viable pCan 1 cell colonies.

Additional studies showed that A1874 concentrationdependently suppressed BrdU incorporation in pCan1 cells (Fig. 1c). The percentage of cell nuclei with positive EdU staining was robustly decreased with A1874 (25-500 nM, $48 \mathrm{~h}$ ) treatment (Fig. 1d). These results confirm the anti-proliferative activity of the BRD4degrading PROTAC in pCan1 primary colon cancer cells. As the titration experimental results in Fig. 1a-d showed that $100 \mathrm{nM}$ of A1874 efficiently inhibited pCan1 cell viability and proliferation, this concentration was selected for the additional studies.

By applying the PI-FACS assay, we found that A1874 $(100 \mathrm{nM})$ resulted in cell cycle arrest by increasing the accumulation of pCan 1 cells in G1-phase while reducing S- and G2/M-phase cells. Therefore, A1874-induced G1-S arrest (Fig. 1e). Examining the potential effect of A1874 on cancer cell migration, "Transwell” assay results, Fig. 1f, demonstrated that A1874 (100 nM) inhibited pCan1 cell migration in vitro. Furthermore, pCan1 cell invasion, tested by "Matrigel Transwell" assay, was suppressed by A1874 (Fig. 1g).

We also examined the potential activity of A1874 in other colon cancer cells. Primary colon cancer cells derived from three patients, pCan $2 / 3 / 4$, as well as established HCT116 cells, were tested. In these colon cancer cells, we found that A1874 $(100 \mathrm{nM})$ potently inhibited cell viability, proliferation and migration, tested by CCK-8 (Fig. 1h), nuclear EdU incorporation (Fig. 1i) and "Transwell" (Fig. 1j) assays, respectively. Together, these studies demonstrate that A1874 potently inhibits colon cancer cell growth, proliferation, cell cycle progression, migration, and invasion.

\section{A1874 induces apoptosis activation in colon cancer cells}

As proliferation inhibition and cell cycle arrest can induce cell apoptosis in colon cancer cells ${ }^{15,31,32}$, we tested whether A1874 can provoke cell death and apoptosis. Trypan blue staining assay results, Fig. 2a, confirmed that A1874 dose-dependently induced pCan 1 colon cancer cell death (increased Trypan blue staining). A1874 (100 nM, $24 \mathrm{~h}$ ) significantly increased caspase-3 and caspase-9 activity in pCan 1 cells (Fig. 2b), leaving caspase- 8 activity unaffected (Fig. 2b). Furthermore, cleavage of caspase3 , caspase-9, and poly ADP-ribose polymerase (PARP) was detected in A1874-treated pCan1 cells (Fig. 2c). A1874 also induced mitochondrial depolarization and caused JC-1 green monomers accumulation in the mitochondria of pCan1 cells (Fig. 2d). These results confirm the activation of mitochondrial apoptosis cascade can-36 $^{33}$ following A1874 treatment (Fig. 2b-d). Further, A1874 $(100 \mathrm{nM})$ induced apoptosis activation in pCan1 cells, causing increased ratios of TUNEL-positive nuclei (Fig. 2e) and Annexin V-positive cells (Fig. 2f).

Significantly, the caspase- 3 inhibitor z-DEVD-fmk and the pan caspase inhibitor $\mathrm{z}$-VAD-fmk largely inhibited A1874 (100 nM)-induced decreased viability (CCK-8 OD) (Fig. 2g), cell death (Trypan blue staining assay, Fig. 2h), and apoptosis activation (nuclear TUNEL staining assay, Fig. 2i). Therefore, apoptosis activation appears to be the primary mechanism of A1874-induced cytotoxicity against pCan1 cells.

In other primary colon cancer cells (pCan2/3/4) and established HCT116 cells, A1874 (100 nM) treatment 


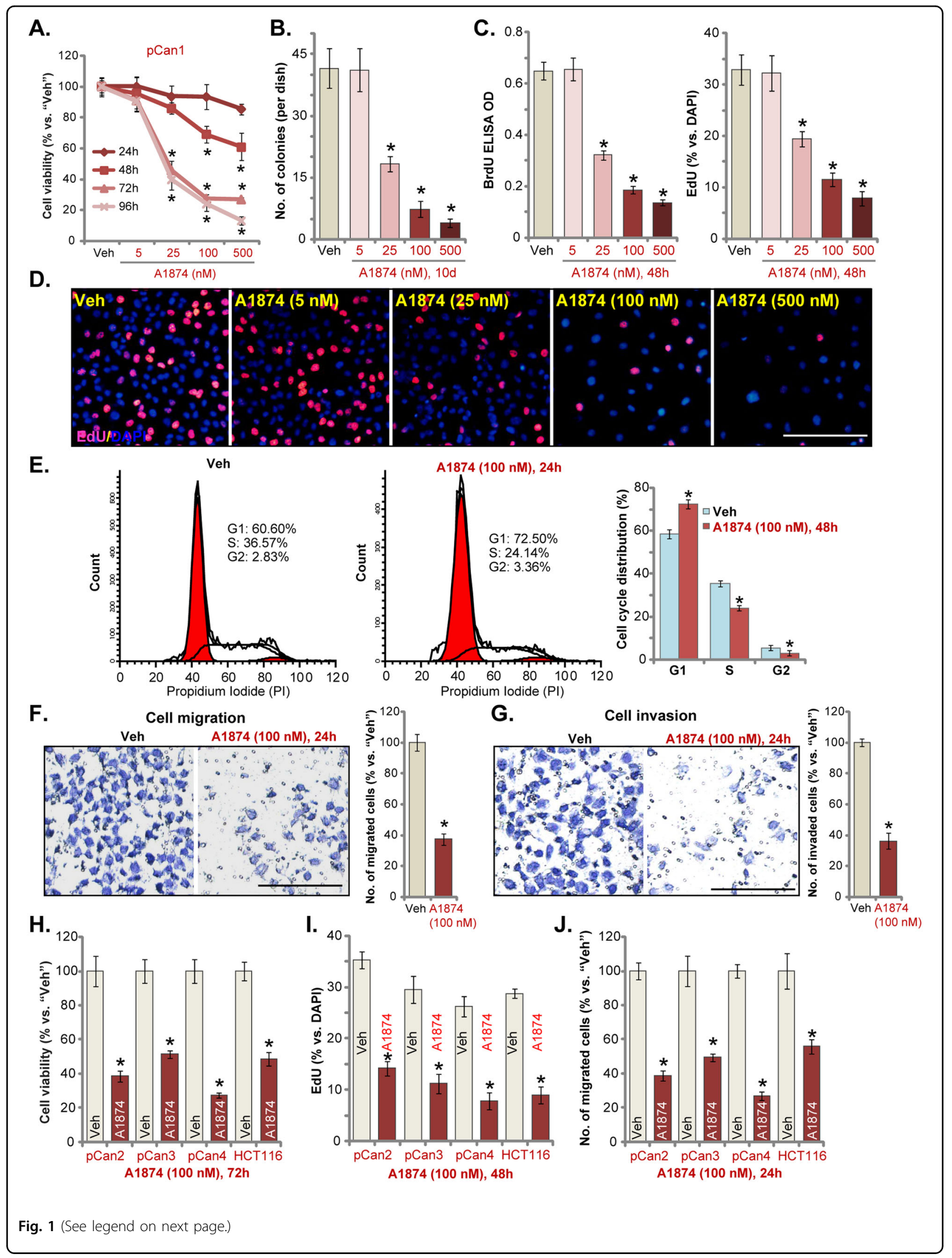


(see figure on previous page)

Fig. 1 A1874 inhibits colon cancer cell growth, proliferation, cell cycle progression, migration and invasion. The primary human colon cancer cells, pCan1/2/3/4 (derived from different colon cancer patients) (a-j) or established HCT116 cells $(\mathbf{h}-\mathbf{j})$ were treated with applied concentration of A1874 (5-500 nM) or the vehicle control ("Veh," 0.2\% of DMSO). Cells were further cultured in complete medium for applied time periods, then cell viability (CCK-8 OD, a and h), colony formation (b) and cell proliferation (BrdU ELISA OD and nuclear EdU incorporation ratio, $\mathbf{c}$, $\mathbf{d}$ and $\mathbf{i}$ ) as well as cell cycle progression (PI-FACS, e), cell migration $(\mathbf{f}, \mathbf{j})$ and invasion $(\mathbf{g})$ were tested by the listed assays. Data were presented as mean \pm standard deviation (SD, $n=5)$. * $P<0.05$ vs. "Veh" cells. Experiments in this figure were repeated three times, and similar results were obtained. Bar $=100 \mu m$ $(\mathbf{d}, \mathbf{f}, \mathbf{g})$.

robustly increased caspase-3 activity (Fig. $2 \mathrm{j}$ ) and TUNELpositive nuclear ratio (Fig. 2k), indicating apoptosis activation. However, in the primary colon epithelial cells ("pEpi1/2," from two donors $\left.{ }^{18}\right)$, A1874 (100 nM) treatment failed to decrease cell viability (Fig. 2l) and induce apoptosis activation (Fig. 2m), indicating a cancer cellspecific effect by the BRD4-degrading PROTAC.

\section{A1874-induced anti-colon cancer cell activity is not solely dependent on BRD4 protein degradation}

Because $\mathrm{A} 1874$ is a novel BRD4-degrading PROTAC ${ }^{14}$, we tested its effect on BRD4 signaling in colon cancer cells. In primary human colon cancer cells, pCan 1 and pCan2, A1874 (100 nM) treatment led to robust degradation of BRD4 protein (Fig. 3a) without affecting BRD4 mRNA expression (Fig. 3b). Furthermore, mRNA and protein expression of BRD4-dependent genes, including $c$ $\mathrm{Myc}, \mathrm{Bcl}-2$, and cyclin D1, was significantly decreased in A1874-treated colon cancer cells (Fig. 3a, b). We then compared the anti-colon cancer cell activity of A1874 with other known BRD4-BET inhibitors, including JQ1 $1^{37,38}$, CPI203 ${ }^{29,39}$, and I-BET726 $6^{40-42}$. In pCan1 cells and pCan2 cells, A1874-induced viability (CCK-8 OD) reduction (Fig. 3c), and cell apoptosis (nuclear TUNEL staining assay, Fig. 3d) were significantly more potent than JQ1, CPI203, and I-BET726. These known inhibitors were utilized at even higher concentrations than A1874. These results suggest that A1874-induced anti-colon cancer cell activity might not be solely dependent on BRD4 protein degradation.

To explore whether A1874 acts solely to promote BRD4 protein degradation, a mutant BRD4 expression construct ["BRD4 (Mut)"], with a mutation at the MDM2's binding site $^{14}$, was stably transduced into pCan1 cells. Western blotting assay results, Fig. 3e, demonstrated that BRD4 and c-Myc protein expression was restored by the BRD4 (Mut) construct even after A1874 treatment. However, the BRD4 (Mut) only partially inhibited A1874 (100 nM)induced cell death (Trypan blue ratio increase, Fig. 3f) and apoptosis (nuclear TUNEL staining assay, Fig. $3 g$ ). These studies support the existence of BRD4-independent mechanisms responsible for A1874-induced cytotoxicity in colon cancer cells.

To further support our hypothesis, we found that CRISPR/Cas9-induced BRD4 KO resulted in c-Myc downregulation (Fig. 3h), causing pCan1 cell death (Fig. 3i) and apoptosis (Fig. 3j). In BRD4-KO cells, A1874 $(100 \mathrm{nM})$ was able to induce further cell death (Fig. 3i) and apoptosis (Fig. 3j). Thus, in addition to BRD4 protein degradation, parallel cell death mechanisms are also responsible for A1874-induced anti-colon cancer cell activity.

\section{A1874 induces p53 protein stabilization and oxidative injury in colon cancer cells}

Possible A1874-induced parallel cell death mechanisms include p53 protein stabilization and oxidative injury. A1874 is a novel BRD4-targeting MDM2-based PROTAC, reported to stabilize p53 protein ${ }^{14}$. In the primary colon cancer cells, pCan1 and pCan2, treatment with A1874 $(100 \mathrm{nM}, 8 \mathrm{~h})$ induced robust p53 protein elevation (Fig. 4a), whereas expression of p53 mRNA was unchanged (Fig. 4b). Interestingly, A1874-induced significant oxidative injury in colon cancer cells, increasing CellROX fluorescence intensity ${ }^{43}$ in pCan1 and pCan2 cells (Fig. 4c). A1874-induced oxidative stress in colon cancer cells was also indicated by the GSH/GSSG ratio reduction (Fig. 4d) and ssDNA accumulation (Fig. 4e, DNA breaks).

To study whether p53 protein stabilization and oxidative injury participate in A1874-induced anti-colon cancer cell activity, the antioxidant NAC and the p53 inhibitor pifithrin- $\alpha^{44,45}$ were applied. As shown, A1874 (100 nM)induced viability (CCK-8 OD) reduction was inhibited by NAC and pifithrin- $\alpha$ in pCan 1 cells (Fig. 4f). Furthermore, NAC and pifithrin- $\alpha$ mitigated A1874-induced pCan1 cell apoptosis (nuclear TUNEL staining assay, Fig. 4g). Significantly, CRISPR/Cas9-induced BRD4 KO (see Fig. 3) failed to promote p53 protein upregulation (Fig. 4h) and ROS production (CellROX intensity, Fig. 4i) in pCan1 cells. These results demonstrate that p53 stabilization and oxidative injury act independently of BRD4 protein degradation to participate in A1874induced anti-colon cancer cell activity.

\section{A1874 oral administration inhibits colon cancer xenograft growth in SCID mice}

In order to study the potential anticancer activity of A1874 in vivo, pCan1 colon cancer cells were s.c. injected into the flanks of SCID mice. Within three weeks, colon cancer xenografts were established with tumor volumes 


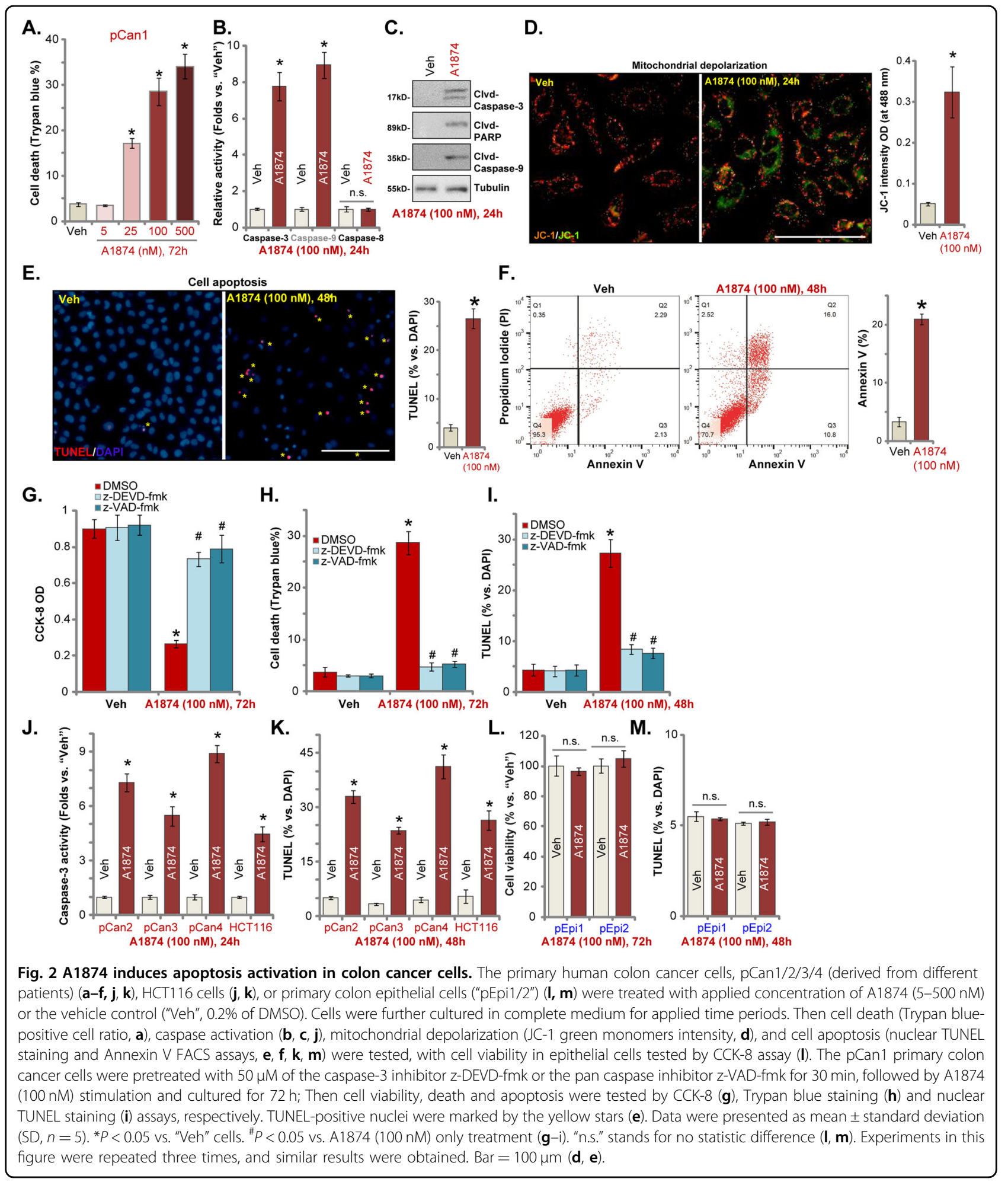

close to $100 \mathrm{~mm}^{3}$ (Day-0/D0). By recording tumor growth curve, we demonstrated that A1874 oral administration ( $20 \mathrm{mg} / \mathrm{kg}$, daily, 21 days) potently inhibited colon cancer xenograft growth in SCID mice (Fig. 5a). Calculating the estimated daily tumor growth, using the formula: (Tumor volume at D42-Tumor volume at D0)/42 (days), we found that colon cancer xenograft growth was largely inhibited in A1874-treated mice (Fig. 5b). Tumors from the two groups were isolated and weighted individually at experimental Day-42 (D42). Xenograft tumors with 


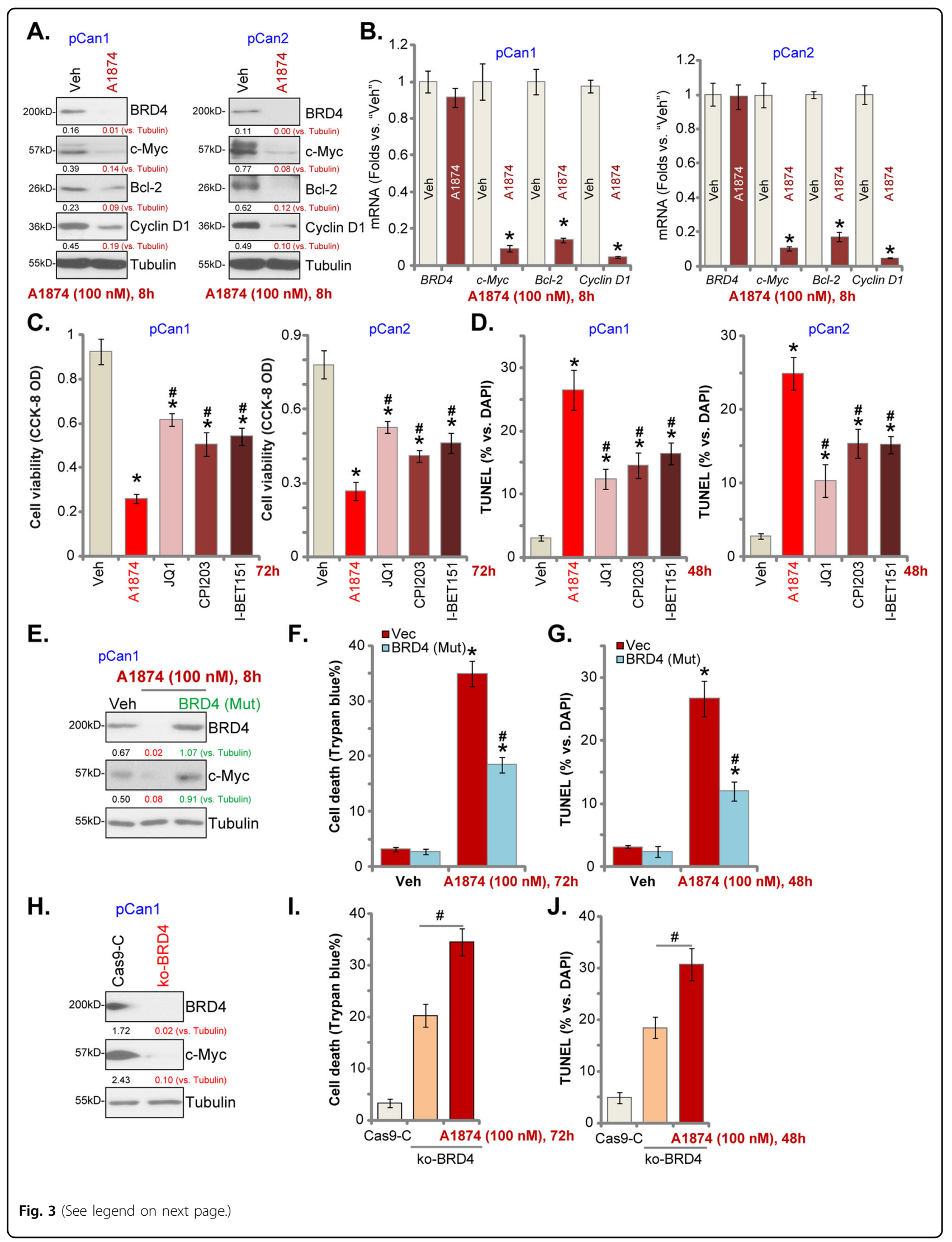


(see figure on previous page)

Fig. 3 A1874-induced anti-colon cancer cell activity is not solely dependent on BRD4 protein degradation. The primary human colon cancer cells, pCan1 and pCan2, were treated with A1874 (100 nM) or the vehicle control ("Veh", 0.2\% of DMSO).Cells were further cultured in complete medium for applied time periods, and then expression of listed proteins (a) and mRNAs (b) were shown. The primary human colon cancer cells, pCan1 and pCan2, were treated with A1874 (100 nM), JQ1 (500 nM), I-BET726 (200 nM), CPI203 (500 nM) or the vehicle control ("Veh," 0.2\% of DMSO) and further cultured in complete medium for applied time periods, and then cell viability and apoptosis were tested by CCK-8 (c) and nuclear TUNEL staining (d) assays, respectively. Stable pCan1 cells with the mutant BRD4 expression construct ["BRD4 (Mut)"] or the empty vector ("Vec") were treated with or without A1874 (100 nM). Cells were then further cultured in complete medium for applied time periods, and expression of listed proteins was shown (e); Cell death and apoptosis were tested by Trypan blue staining (f) and nuclear TUNEL staining (g) assays, respectively. The stable pCan1 cells with CRISPR/Cas9-BRD4-KO-GFP construct ("ko-BRD4" cells) were treated with or without A1874 (100 nM). The control cells with CRISPR/Cas9 empty vector ("Cas9-C") were left untreated; Cells were further cultured in complete medium for applied time periods, and then expression of listed proteins was shown (h); Cell death and apoptosis were tested by Trypan blue staining (i) and nuclear TUNEL staining (j) assays, respectively. Expression of listed proteins was quantified and normalized to the loading control $(\mathbf{a}, \mathbf{e}, \mathbf{h})$. Data were presented as mean \pm standard deviation (SD, $n=5) .{ }^{*} P<0.05$ vs. "Veh" cells. ${ }^{*} P<0.05$ vs. A1874 treatment $(\mathbf{c}, \mathbf{d}) .{ }^{~} P<0.05$ vs. "Vec" cells $(\mathbf{f}, \mathbf{g}) .{ }^{*} P<0.05$ (I and $\left.\mathbf{J}\right)$. Experiments in this figure were repeated three times, and similar results were obtained.

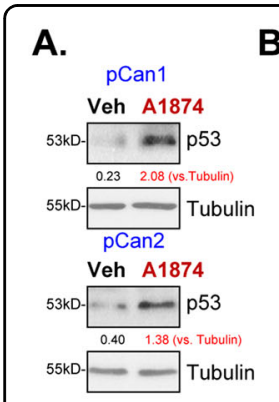

A1874 (100 nM),8h

D.

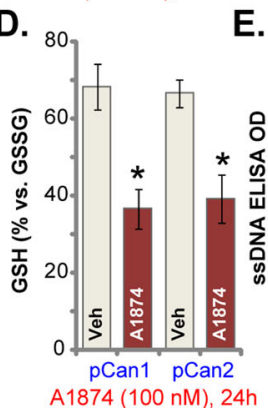

B.

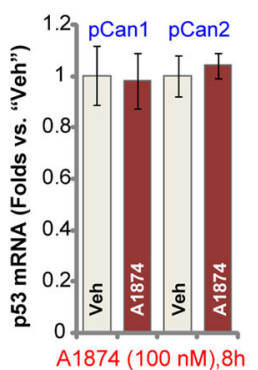

E. $F$

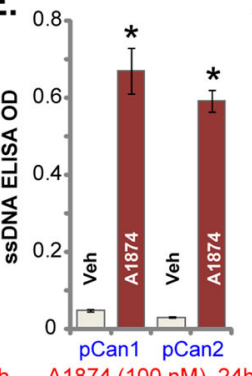

C.

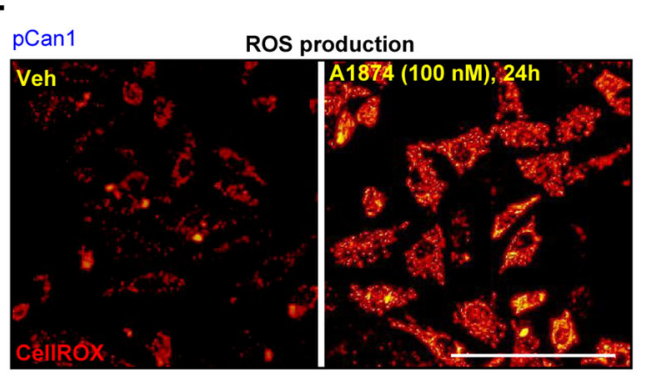

F.

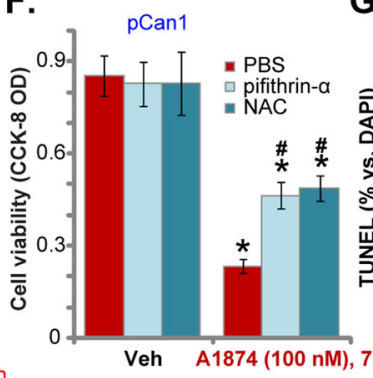

G.

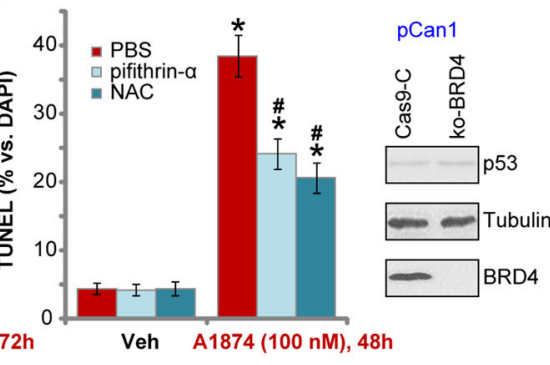

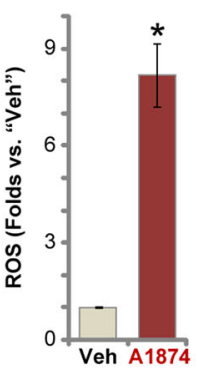

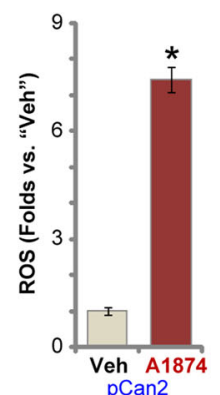

I.

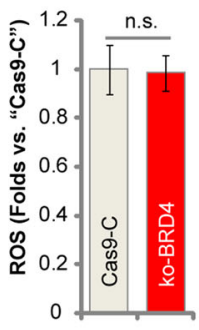

Fig. 4 A1874 induces p53 protein stabilization and oxidative injury in colon cancer cells. The primary human colon cancer cells, pCan1 and pCan2, were treated with A1874 (100 nM) or the vehicle control ("Veh", $0.2 \%$ of DMSO). Cells were further cultured in complete medium for applied time periods, and then expressions of p53 protein (a) and mRNA (b) were shown; The CellROX intensity (c), the GSH/GSSG ratio (d) and the single strand DNA (ssDNA) contents (e) were tested as well. The pCan1 cells were pretreated for $1 \mathrm{~h}$ with the antioxidant N-acetyl-cysteine (NAC, $400 \mu \mathrm{M})$ or the p53 inhibitor pifithrin-a $(10 \mu \mathrm{M})$, followed by A1874 (100 nM) stimulation for another 48-72 h.Then cell viability was tested by CCK-8 assay (f), with cell apoptosis examined by nuclear TUNEL staining assay (g). Stable pCan1 cells with CRISPR/Cas9-BRD4-KO-GFP construct ("ko-BRD4" cells) or control cells with CRISPR/Cas9 empty vector ("Cas9-C") were cultured for $24 \mathrm{~h}$, and then expression of listed proteins (h) and ROS contents (CellROX intensity, i) were tested. Expression of listed proteins was quantified and normalized to the loading control (a). Data were presented as mean \pm standard deviation (SD, $n=5)$. ${ }^{*} P<0.05$ vs. "Veh" cells. ${ }^{*} P<0.05$ vs. A1874 treatment $(\mathbf{f}, \mathbf{g})$. Experiments in this figure were repeated three times, and similar results were obtained. Bar $=100 \mu \mathrm{m}$ (c). "n.s." stands for no statistic difference (i).

A1874 administration were significantly lighter than those of vehicle control mice (Fig. 5c). Mouse body weights were not significantly different between A1874-treated and vehicle control mice (Fig. 5d), and no noticeable toxicity was observed in the mice. These results show that oral administration of $\mathrm{A} 1874$ is able to inhibit colon cancer xenograft growth in SCID mice.
At treatment D7, one tumor from each group was isolated, and tumors were homogenized. Western blotting analyses showed that protein expression of BRD4, c-Myc, Bcl-2 and cyclin D1, were significantly decreased in A1874-treated tumor tissues (Fig. 5e), where caspase-3 and PARP cleavage was detected (Fig. 5f). Furthermore, p53 protein elevation was observed in xenograft tissues 

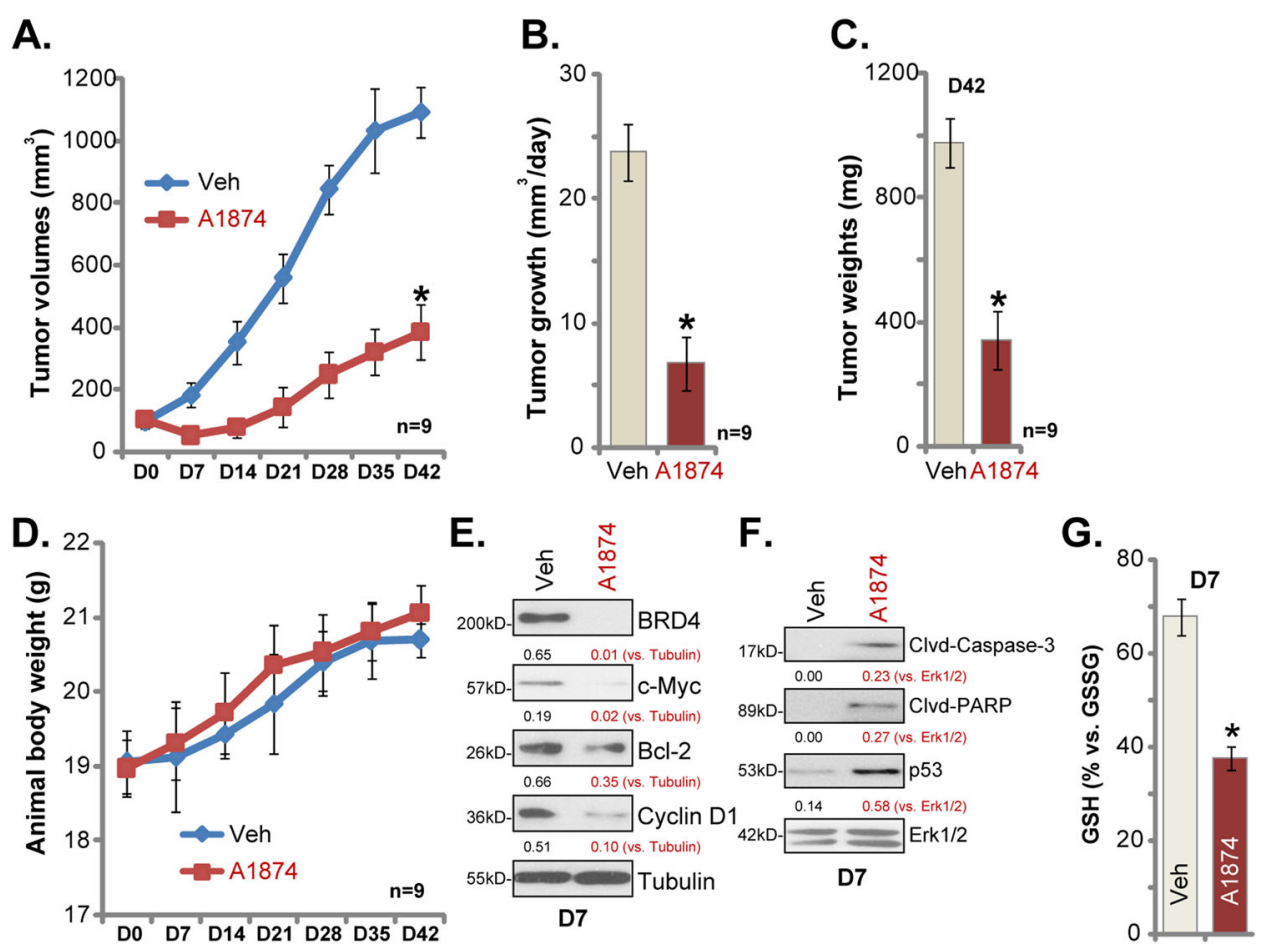

Fig. 5 A1874 oral administration inhibits colon cancer xenograft growth in SCID mice. The SCID mice bearing pCan1 colon cancer xenografts were treated with A1874 (20 mg/kg body weight, oral administration, daily for 21 days) or the vehicle control ("Veh"); Tumor volumes (a) and mice body weights (d) were recorded weekly. The estimated daily tumor growth was calculated by using the described formula (b). At Day-42/D42, tumors of the two groups were isolated and weighted (c). At treatment Day7 (D7), one tumor of each group was isolated, and tumors were homogenized. Expression of the listed proteins in tumor tissue lysates was tested (e, f). The GSH/GSSG ratio in tumor tissue lysates was examined (g). Expression of listed proteins was quantified and normalized to the loading control (e, $\mathbf{f})$. Data were presented as mean \pm standard deviation (SD). * $P<0.05$ vs. "Veh" group.

with A1874 administration (Fig. 5f). Additionally, the GSH/GSSG ratio was decreased in A1874-treated xenograft tissue lysates, indicating oxidative stress (Fig. $5 \mathrm{~g}$ ). Therefore, in line with in vitro findings, A1874 was able to induce BRD4 protein degradation, apoptosis activation, p53 elevation and oxidative stress in colon cancer xenografts.

\section{Discussion}

BRD4, a gene that is overexpressed in different human cancers, is associated with carcinogenesis, tumorigenesis, and progression of human malignancies. It is emerging as a promising therapeutic target ${ }^{8,46,47}$. The development and optimization of BRD4 small-molecule inhibitors as novel cancer therapeutics are currently a major focus of cancer research ${ }^{8,46,47}$. BRD4 binds directly to the acetylated histones to promote transcription and expression of multiple oncogenic genes, including $\mathrm{c}-\mathrm{Myc}$ and several others $^{8,46,47}$. BRD4 also acts as an associated factor of P$\mathrm{TEFb}$, stimulating RNA polymerase II-dependent transcription and cell cycle progression ${ }^{10,47,48}$.

BRD4 overexpression is detected in colorectal cancer $(\mathrm{CRC})^{12}$. Hu et al., showed that MS417, a BRD4 blocker, potently inhibited CRC growth, epithelial-tomesenchymal transition progression and metastasis ${ }^{12}$. Togel et al. demonstrated that BRD4 blockage downregulated c-Myc and inhibited CRC cell proliferation, which could be further augmented by targeting WNT or MAPK signaling ${ }^{49}$.

To date more than ten BET inhibitors have advanced to early stage clinical trials for patients with different types of cancer $^{11,13}$. However, BRD4 inhibitors were found to only exert limited antitumor activity in patients ${ }^{11,13}$. One possibility is that BRD4 inhibition induces feedback upregulation of the BRD4 protein, leading to modest antiproliferative ability and minor apoptotic induction ${ }^{8,11,13}$. Therefore, a new therapeutic approach is urgently needed to target BRD4 and other BET proteins ${ }^{8,11,13}$.

The BRD4 PROTACs have two covalently linked protein-binding domains: one capable of engaging an E3 ubiquitin ligase, and the other binding to BRD4 protein for ubiquitination-mediated degradation ${ }^{50}$. These compounds differ significantly from small-molecular BRD4 inhibitors in their cellular potency, phenotypic effects, pharmacokinetic kinetics and potential toxicity profiles ${ }^{50}$. A1874 is a first-in-class BRD4-targeting MDM2-based 
PROTAC $^{14}$. Studies have shown that it results in robust and sustained BRD4 degradation ${ }^{14}$. Furthermore, A1874 increases p53 stabilization and protein levels in a dosedependent manner ${ }^{14}$.

Here, in primary colon cancer cells and established HCT116 cells, A1874 potently inhibited cell viability, growth, proliferation and cell cycle progression, as well as cell migration and invasion. Furthermore, the BRD4degrading PROTAC induced significant apoptosis activation in primary and established colon cancer cells. At the molecular level, A1874 is able to induce BRD4 protein degradation and the downregulation of BRD-dependent genes (c-Myc, Bcl-2 and cyclin D1) in colon cancer cells.

Although A1874-induced robust and potent BRD4 protein degradation, A1874-induced anti-colon cancer cell activity was not solely dependent on BRD4 degradation. First, A1874 was significantly more potent than other known BRD4/BET inhibitors (JQ1, CPI203, IBET151) at inducing colon cancer cell apoptosis. Second, restoring BRD4 expression by the BRD4 (Mut) construct only partially inhibited A1874-induced anti-colon cancer cell activity. Third, the novel MDM2-recruiting PROTAC remained cytotoxic in the BRD4-KO colon cancer cells. A1874-induced p53 protein stabilization and oxidative stress in colon cancer cells, two actions that are independent of BRD4 depletion. Conversely, the antioxidant NAC and the p53 inhibitor pifithrin- $\alpha$ attenuated A1874induced colon cancer cell apoptosis. Therefore A1874 acts via both BRD4-dependent and BRD4-independent (p53 stabilization and ROS production) mechanisms, providing an explanation for its superior anticancer activity against colon cancer cells.

Colon cancer and other CRC are among the third most common type of cancer, accounting for around $10 \%$ of all malignancies $^{3,51}$. In 2018, there are 1.09 million new cases and 551,000 CRC deaths (mainly colon cancer). The 5year survival rate of CRC in the United States is close to $65 \%^{3,51}$. Molecularly targeted therapies are the current focus of research for colon cancer ${ }^{52,53}$. Here we report that oral administration of a single dose of A1874 potently inhibits colon cancer xenograft growth in SCID mice. These results demonstrate that this novel compound is a promising therapeutic to treat colon cancer.

\section{Acknowledgements \\ The present study was supported by the grant for Key Young Talents of Medicine in Jiangsu (QNRC2016250), Gusu medical talent project (GSWS2019026 and GSWS2019025), and Foundation of tumor clinical and basic research team of Affiliated Kunshan Hospital of Jiangsu University (KYC005).}

\footnotetext{
Author details

'Department of Hepatobiliary Surgery, Suzhou Municipal Hospital Affiliated to Nanjing Medical University, Suzhou, China. ${ }^{2}$ The Child Health Care Department, Suzhou Ninth People's Hospital, Suzhou, China. ${ }^{3}$ Department of Oncology, The Affiliated Zhangjiagang Hospital of Soochow University, Suzhou, China.

${ }^{4}$ Trauma center, Affiliated Hospital of Yangzhou University, Yangzhou, China.
}

${ }^{5}$ Department of Radiotherapy and Oncology, Affiliated Kunshan Hospital of Jiangsu University, Kunshan, China. ${ }^{6}$ Department of General Surgery, The Affiliated Zhangjiagang Hospital of Soochow University, Suzhou, China

Conflict of interest

The authors declare that they have no conflict of interest.

\section{Publisher's note}

Springer Nature remains neutral with regard to jurisdictional claims in published maps and institutional affiliations.

Received: 28 July 2020 Revised: 4 September 2020 Accepted: 7 September 2020

Published online: 25 September 2020

\section{References}

1. Palma, S., Zwenger, A. O., Croce, M. V., Abba, M. C. \& Lacunza, E. From molecular biology to clinical trials: toward personalized colorectal cancer therapy. Clin. Colorectal Cancer 15, 104-115 (2016).

2. Hubbard, J. M. \& Grothey, A. Colorectal cancer in 2014: progress in defining first-line and maintenance therapies. Nat. Rev. Clin. Oncol. 12, 73-74 (2015).

3. Siegel, R. L., Miller, K. D. \& Jemal, A. Cancer statistics, 2018. CA Cancer J. Clin. 68, 7-30 (2018).

4. Siegel, R. L., Miller, K. D. \& Jemal, A. Cancer Statistics, 2017. CA Cancer J. Clin. 67, 7-30 (2017).

5. Sugarbaker, P. H. Colorectal cancer: prevention and management of metastatic disease. Biomed. Res. Int. 2014, 782890 (2014).

6. Schmoll, H. J. \& Stein, A. Colorectal cancer in 2013: towards improved drugs, combinations and patient selection. Nat. Rev. Clin. Oncol. 11, 79-80 (2014).

7. Wu, X. et al. Inhibition of BRD4 suppresses cell proliferation and induces apoptosis in renal cell carcinoma. Cell Physiol. Biochem. 41, 1947-1956 (2017).

8. White, M. E., Fenger, J. M. \& Carson, W. E. III Emerging roles of and therapeutic strategies targeting BRD4 in cancer. Cell Immunol. 337, 48-53 (2019).

9. Devaiah, B. N. \& Singer, D. S. Two faces of brd4: mitotic bookmark and transcriptional lynchpin. Transcription 4, 13-17 (2013).

10. Wu, S. Y. \& Chiang, C. M. The double bromodomain-containing chromatin adaptor Brd4 and transcriptional regulation. J. Biol. Chem. 282, 13141-13145 (2007).

11. Hajmiza, A. et al. BET family protein BRD4: an emerging actor in NFkappaB signaling in inflammation and cancer. Biomedicines. 6 (2018).

12. Hu, Y. et al. BRD4 inhibitor inhibits colorectal cancer growth and metastasis. Int J. Mol. Sci. 16, 1928-1948 (2015)

13. Fu, L. L. et al. Inhibition of BET bromodomains as a therapeutic strategy for cancer drug discovery. Oncotarget 6, 5501-5516 (2015).

14. Hines, J., Lartigue, S., Dong, H., Qian, Y. \& Crews, C. M. MDM2-recruiting PROTAC offers superior, synergistic antiproliferative activity via simultaneous degradation of BRD4 and stabilization of p53. Cancer Res. 79, 251-262 (2019).

15. Chen, M. B. et al. Activation of AMP-activated protein kinase (AMPK) mediates plumbagin-induced apoptosis and growth inhibition in cultured human colon cancer cells. Cell Signal. 25, 1993-2002 (2013).

16. Lu, P. H. et al. Aqueous Oldenlandia diffusa extracts inhibits colorectal cancer cells via activating AMP-activated protein kinase signalings. Oncotarget $\mathbf{7}$ 45889-45900 (2016).

17. $L i, C$. et al. The preclinical evaluation of the dual mTORC1/2 inhibitor INK-128 as a potential anti-colorectal cancer agent. Cancer Biol. Ther. 16, 34-42 (2015).

18. Li, G. et al. Ninjurin 2 overexpression promotes human colorectal cancer cell growth in vitro and in vivo. Aging. 11, 8526-8541 (2019).

19. Wang, S. S. et al. Triptonide inhibits human nasopharyngeal carcinoma cell growth via disrupting Lnc-RNA THOR-IGF2BP1 signaling. Cancer Lett. $\mathbf{4 4 3}$ 13-24 (2019).

20. Yang, L. et al. C6 ceramide dramatically enhances docetaxel-induced growth inhibition and apoptosis in cultured breast cancer cells: a mechanism study. Exp. Cell Res. 332, 47-59 (2015).

21. Zhou, L. N., Li, P., Cai, S., Li, G. \& Liu, F. Ninjurin2 overexpression promotes glioma cell growth. Aging 11, 11136-11147 (2019). 
22. LV, Y. et al. Overexpression of lymphocyte antigen 6 complex, locus $E$ in gastric cancer promotes cancer cell growth and metastasis. Cell Physiol. Biochem. 45, 1219-1229 (2018).

23. Zheng, B. et al. Pre-clinical evaluation of AZD-2014, a novel mTORC1/2 dual inhibitor, against renal cell carcinoma. Cancer Lett. 357, 468-475 (2015).

24. Brooks, M. M., Neelam, S., Fudala, R., Gryczynski, I. \& Cammarata, P. R. Lenticular mitoprotection. Part A: monitoring mitochondrial depolarization with JC-1 and artifactual fluorescence by the glycogen synthase kinase-3beta inhibitor, SB216763. Mol. Vis. 19, 1406-1412 (2013).

25. Cao, C. et al. Impairment of TrkB-PSD-95 signaling in Angelman syndrome. PLOS Biol. 11, e1001478 (2013).

26. Ye, X., Xie, J., Huang, H. \& Deng, Z. Knockdown of MAGEA6 activates AMPactivated protein kinase (AMPK) signaling to inhibit human renal cell carcinoma cells. Cell Physiol. Biochem. 45, 1205-1218 (2018).

27. Zheng, J. et al. MicroRNA-4651 targets bromodomain-containing protein 4 to inhibit non-small cell lung cancer cell progression. Cancer Lett. 476, 129-139 (2020).

28. Zitka, O. et al. Redox status expressed as GSH:GSSG ratio as a marker for oxidative stress in paediatric tumour patients. Oncol. Lett. 4, 1247-1253 (2012)

29. Xiang, T. et al. Bromodomain protein BRD4 promotes cell proliferation in skin squamous cell carcinoma. Cell Signal. 42, 106-113 (2018).

30. Chen, M. B. et al. Itraconazole-induced inhibition on human esophageal cancer cell growth requires AMPK activation. Mol. Cancer Ther. 17, 1229-1239 (2018).

31. Su, C. et al. Targeting p38gamma to inhibit human colorectal cancer cell progression. Biochem. Biophys. Res. Commun. 517, 172-179 (2019).

32. $\mathrm{Xu}, \mathrm{W}$., Xu, B., Yao, Y., Yu, X. \& Shen, J. The novel HDAC inhibitor AR-42-induced anti-colon cancer cell activity is associated with ceramide production. Biochem. Biophys. Res. Commun. 463, 545-550 (2015).

33. Fu, M., Wan, F., Li, Z. \& Zhang, F. 4SC-202 activates ASK1-dependent mitochondrial apoptosis pathway to inhibit hepatocellular carcinoma cells. Biochem. Biophys. Res. Commun. 471, 267-273 (2016).

34. Fu, M., Shi, W., Li, Z. \& Liu, H. Activation of mPTP-dependent mitochondrial apoptosis pathway by a novel pan HDAC inhibitor resminostat in hepatocellular carcinoma cells. Biochem. Biophys. Res. Commun. 477, 527-533 (2016).

35. Riedl, S. J. \& Shi, Y. Molecular mechanisms of caspase regulation during apoptosis. Nat. Rev. Mol. Cell Biol. 5, 897-907 (2004).

36. Chen, M. \& Wang, J. Initiator caspases in apoptosis signaling pathways. Apoptosis 7, 313-319 (2002).

37. Lee, D. H. et al. Synergistic effect of JQ1 and rapamycin for treatment of human osteosarcoma. Int. J. Cancer 136, 2055-2064 (2015).
38. Korb, E., Herre, M., Zucker-Scharff, I., Darnell, R. B. \& Allis, C. D. BET protein Brd4 activates transcription in neurons and BET inhibitor Jq1 blocks memory in mice. Nat. Neurosci. 18, 1464-1473 (2015).

39. Wong, C. et al. The bromodomain and extra-terminal inhibitor CPI203 enhances the antiproliferative effects of rapamycin on human neuroendocrine tumors. Cell Death Dis. 5, e1450 (2014).

40. Liu, Z. et al. I-BET726 suppresses human skin squamous cell carcinoma cell growth in vitro and in vivo. Cell Death Dis. 11, 318 (2020).

41. Gosmini, R. et al. The discovery of I-BET726 (GSK1324726A), a potent tetrahydroquinoline ApoA1 up-regulator and selective BET bromodomain inhibitor. J. Med. Chem. 57, 8111-8131 (2014).

42. Wyce, A. et al. BET inhibition silences expression of MYCN and BCL2 and induces cytotoxicity in neuroblastoma tumor models. PLOS ONE 8, e72967 (2013).

43. Celeghini, E. C. C., et al. Efficiency of CellROX deep red((R)) and CellROX orange ((R)) fluorescent probes in identifying reactive oxygen species in sperm samples from high and low fertility bulls. Anim. Biotechnol. 1-7 (2019).

44. Bu, H. Q. et al. Oridonin induces apoptosis in SW1990 pancreatic cancer cells via p53- and caspase-dependent induction of p38 MAPK. Oncol. Rep. 31 975-982 (2014).

45. Komarov, P. G. et al. A chemical inhibitor of p53 that protects mice from the side effects of cancer therapy. Science 285, 1733-1737 (1999).

46. Iftner, T., Haedicke-Jarboui, J., Wu, S. Y. \& Chiang, C. M. Involvement of Brd4 in different steps of the papillomavirus life cycle. Virus Res. 231, 76-82 (2017)

47. Chen, R., Yik, J. H., Lew, Q. J. \& Chao, S. H. Brd4 and HEXIM1: multiple roles in PTEFb regulation and cancer. Biomed. Res. Int. 2014, 232870 (2014).

48. Jang, M. K. et al. The bromodomain protein Brd4 is a positive regulatory component of P-TEFb and stimulates RNA polymerase II-dependent transcription. Mol. Cell. 19, 523-534 (2005).

49. Togel, L. et al. Dual targeting of bromodomain and extraterminal domain proteins, and WNT or MAPK signaling, inhibits c-MYC expression and proliferation of colorectal cancer cells. Mol. Cancer Ther. 15, 1217-1226 (2016).

50. Yang, C. Y., Qin, C., Bai, L. \& Wang, S. Small-molecule PROTAC degraders of the bromodomain and extra terminal (BET) proteins-a review. Drug Discov. Today Technol. 31, 43-51 (2019).

51. Siegel, R. L., Miller, K. D. \& Jemal, A. Cancer statistics, 2019. CA Cancer J. Clin. 69, 7-34 (2019).

52. Chu, E. An update on the current and emerging targeted agents in metastatic colorectal cancer. Clin. Colorectal Cancer 11, 1-13 (2012).

53. Lea, M. A. Recently identified and potential targets for colon cancer treatment. Future Oncol. 6, 993-1002 (2010). 